\title{
Wenn sich Menschen ändern - Veränderungsprozesse in der Psychotherapie und ihre Beziehung zu spezifischen Therapietechniken und allgemeinen Wirkfaktoren
}

\author{
Michael J. Lambert ${ }^{\mathrm{a}} \quad$ Maria Kleinstäuber ${ }^{\mathrm{b}}$ \\ a Department of Psychology, Brigham Young University, Provo, UT, USA; \\ ${ }^{\text {b } K l i n i s c h e ~ P s y c h o l o g i e ~ u n d ~ P s y c h o t h e r a p i e, ~ P h i l i p p s-U n i v e r s i t a ̈ t, ~ M a r b u r g, ~ D e u t s c h l a n d ~}$
}

\section{Schlüsselwörter \\ Placebo in der Psychotherapie - Early response . \\ Behandlungsergebnisse · Allgemeine Wirkfaktoren}

\section{Zusammenfassung}

Erkenntnisse diverser Studien legen nahe, dass PlaceboKontrollgruppen mehr von Psychotherapie profitieren als Kontrollgruppen ohne jegliche Behandlung, jedoch weniger als Patienten, die eine theoriegeleitete Behandlung erhalten. Die vorliegende Auswertung der Befunde vergleichender Ergebnisstudien - zum einen solche, die Ergebnisse von Patienten, die von auszubildenden/paraprofessionellen versus professionellen Therapeuten behandelt wurden, gegenüberstellen und zum anderen solche, die eine Symptomverbesserung in der Anfangsphase der Behandlung (,early response' bzw. frühe Therapie-Response) in einem beträchtlichen Anteil der Stichprobe zeigen - legt nahe, dass den allgemeinen Wirkfaktoren von Psychotherapie bedeutende Effekte zukommen. Das Phänomen der frühen Therapie-Response stellt dabei eine Herausforderung dar, sowohl für das Verständnis der Effekte spezifischer psychotherapeutischer Ansätze als auch der ursächlichen Effekte spezifischer therapeutischer Techniken in der Behandlung bestimmter Erkrankungen. Die Behandlungsergebnisse sind dabei am ehesten als komplexes Zusammenspiel allgemeiner Wirkfaktoren, wie z.B. der Erwartung des Patienten hinsichtlich einer erfolgreichen Behandlung oder der therapeutischen Beziehung, sowie spezifischer Interventionen zu verstehen.

(c) 2016 S. Karger GmbH, Freiburg

Das englische Original ist online abrufbar unter www.karger.com/doi/10.1159/000442372

\begin{tabular}{ll}
\hline KARGER & ๑ 2016 S. Karger GmbH, Freiburg \\
& $1016-6262 / 16 / 0261-0032 \$ 39.50 / 0$ \\
Fax +497614520714 & Accessible online at: \\
Information@Karger.com & www.karger.com/ver \\
www.karger.com &
\end{tabular}

\author{
Keywords \\ Placebo in psychotherapy - Early dramatic treatment \\ response - Psychotherapy outcome · Common factors
}

\section{Summary}

Evidence is presented demonstrating that placebo control groups benefit more than no-treatment control groups but less than patients who receive theory-driven treatments. Through a brief review of the results of comparative outcome studies, studies which compare patient outcomes for those seen by trainees/paraprofessionals versus professional therapists, and those which show an early response of a sizable portion of patients, a case is made for the powerful effects of common factors in psychotherapy. The early response phenomenon is proposed as yet another challenge to the unique effects of specific psychotherapies and to the wisdom of emphasizing the causative effects of specific techniques in the treatment of specific disorders. Outcome can be best viewed as a complex interaction of common factors such as patient expectancy for a positive outcome, other common factors, such as relationship, and specific techniques. 


\section{Einleitung}

Die wissenschaftliche Evaluierung der Wirksamkeit von Psychotherapie orientiert sich häufig an den Methoden und der Denklehre anderer Bereiche von Wissenschaft und Medizin. Diese Art von Evaluation ist darauf ausgerichtet, die Wirkkomponenten der Psychotherapie zu identifizieren und schließlich mit diesem Wissen unsere Fähigkeiten zu verbessern, um so maximal wirksam psychische Störungen zu behandeln. In diesem Zusammenhang und unter Berücksichtigung der «Theorie der überzeugenden Schlussfolgerung» nach Platt [1964] («theory of strong inference»; über den wiederholt ablaufenden Prozess der Bildung, Überprüfung und systematischen Verwerfung mehrerer miteinander konkurrierender Hypothesen über beobachtete Ursache-WirkungsBeziehungen wird die standhafteste These herauskristallisiert und verfeinert) haben Forscher versucht, die «wirksamen Komponenten» einer effektiven Behandlung zu verstehen.

In der medizinischen Forschung erfolgt die Suche nach Bonafide-Behandlungen häufig in der Form, dass die Effekte eines pharmakologischen Wirkstoffs denen einer pharmakologisch unwirksamen Substanz - einem «Placebo» - gegenübergestellt werden. Die Verwendung einer Placebo-Kontrolle ermöglicht es, die konkurrierende Hypothese, dass die Behandlungseffekte durch die Erwartungen der Patienten erklärt werden könnten, auszuschließen. Diese Gegenüberstellung macht bei medikamentösen Therapien Sinn, bei denen der Behandlungserfolg dem pharmakologischen Wirkstoff und nicht den psychologischen Auswirkungen des Akts, behandelt zu werden bzw. ein Medikament einzunehmen, zugeschrieben werden kann. Der Einsatz von Placebo-Kontrollen im Bereich der Psychotherapieforschung macht weniger Sinn, da der Nutzen von Behandlungen und Placebos von psychologischen Mechanismen abhängt. Viele Wissenschaftler der 1980er-Jahre lehnten das Placebo-Konzept in der Psychotherapieforschung ab, weil es konzeptuell nicht im Einklang mit der Überprüfung der Wirksamkeit psychologischer Verfahren in Bezug auf psychische Probleme steht [z.B. Dush, 1986; Horvath, 1988; Wilkins, 1984]. Nach Rosenthal und Frank [1956] zum Beispiel wird ein Placebo als theoretisch unwirksam definiert. Es ist jedoch nur vom Standpunkt der Theorie aus, die hinter der untersuchten Therapie steht, unwirksam. Entsprechend der Feststellung von Critelli und Neumann [1984] «würde praktisch jede gegenwärtig bewährte Psychotherapie aus Sicht anderer etablierter Heilungstheorien als unwirksam und somit als Placebo angesehen werden» (S. 33). Folglich wurden «Placebos» teilweise auch als «unspezifische» Faktoren bezeichnet [z.B. Oei und Shuttlewood, 1996]. Diese Konzeptualisierung wirft ernstzunehmende Fragen über die Definition des Begriffs «unspezifisch» auf. Wird ein «unspezifischer» Faktor, sobald er als solcher benannt und operationalisiert wird, zu einem spezifischen Faktor und fällt dann nicht mehr in den Bereich eines Placeboeffekts? Wird zum Beispiel eine Variable wie «bedingungslose positive $\mathrm{Zu}$ wendung und emotionale Wärme des Therapeuten» in Abhängigkeit davon, ob sie bzw. ob sie nicht operationalisiert und gemessen wird, zu einem spezifischen bzw. unspezifischen Faktor (d.h. einem Placebo [Bowers und Clum, 1988])?
Als Reaktion auf das häufige Problem, dass in vergleichenden Ergebnisstudien keine Zwischengruppenunterschiede gefunden werden, haben andere Forscher den Begriff «allgemeine Wirkfaktoren» als Ersatz für Termini wie «Placebo» oder «unspezifische Faktoren» vorgeschlagen [Lambert, 2013]. Viele therapeutische Ansätze umfassen allgemeine Wirkkomponenten, die nicht einzigartig für eine bestimmte Therapieform, aber dennoch zu einem gewissen Grad wirksam sind. In diesem Zusammenhang ist der Begriff «allgemeine Wirkfaktoren» nicht gleichbedeutend mit «Placebo», der in Bezug auf Erwartungen von Patienten zu Beginn einer Behandlung viel enger definiert ist. Die Forschung zu Placeboeffekten zielt darauf ab, Heilungserwartungen, die vermutlich mit wirksamen wie auch Scheinbehandlungen einhergehen, als zu einer Verbesserung beitragende Faktoren zu kontrollieren. Im Gegensatz dazu versuchen Kontrollgruppen, in denen allgemeine Wirkfaktoren berücksichtigt werden, eine Vielzahl von vermuteten Ursachen psychotherapeutischen Erfolgs auszuschalten und gleichzeitig den Zusatzeffekt spezifischer Techniken innerhalb eines Psychotherapieverfahrens zu bestimmen. Allgemeine Wirkfaktoren stellen jene Dimensionen des Behandlungssettings (Therapeut, Therapie, Patient) dar, die nicht spezifisch für eine bestimmte therapeutische Technik sind.

Im Rahmen der Forschung zum breitgefächerten Konzept allgemeiner Wirkfaktoren werden nicht allein kausale Mechanismen untersucht wie Verbesserungserwartungen, Zuversicht des Therapeuten und eine therapeutische Beziehung, die durch Vertrauen, Wärme, Verständnis, Akzeptanz, Wohlwollen, Lebenserfahrung und Weisheit geprägt ist. Stattdessen kann sie um einige Mechanismen erweitert werden, die häufig als spezifisch für ein bestimmtes Behandlungsverfahren angesehen werden, tatsächlich jedoch in jeder Form von Psychotherapie zu einem gewissen Grad vorhanden sind, z.B. Katharsis, Exposition mit angstauslösenden Situationen/Reizen, Ermutigung, Risikobereitschaft zu zeigen (z.B. sich mit Situationen zu konfrontieren, die dem Patienten Unbehagen bereiten, anstatt sie zu vermeiden), und Förderung von Bewältigungsbemühungen des Patienten wie z.B. Versuche, neues Verhalten zu erproben und zu üben. Diese umfassende Betrachtungsweise $\mathrm{zu}$ allgemeinen Wirkfaktoren erkennt an, dass zwar spezifische Theorien der Psychotherapie systematische In-vivo- oder In-vitroExpositionen mit angstauslösenden Situationen, Training sozialer Fertigkeiten oder Interpretationen in den Vordergrund stellen können, dass im Endeffekt aber fast alle Therapien Menschen ermutigen, sich mit Dingen, vor denen sie Angst haben, auseinanderzusetzen und diese zu diskutieren bzw. sich angstbesetzten Situationen zu stellen, anstatt sie zu vermeiden. Allgemeine Wirkfaktoren der Psychotherapie - unabhängig davon, als wie unwichtig (theoretisch unwirksam oder trivial) sie aus Sicht einer bestimmten Theorie erachtet werden - sind für fast alle psychologischen Interventionen von zentraler Bedeutung, wenn nicht in der Theorie, dann zumindest in der Praxis. Dies mag erklären, warum sich verschiedene Therapieverfahren hinsichtlich der Rate remittierter Patienten kaum unterscheiden. Viele therapeutische Techniken, die als spezifisch für eine - einem bestimmten Psychotherapieverfahren zugrunde liegende - Theorie angesehen werden, 
kommen in der Tat zu einem gewissen Maß in verschiedenen theoriegeleiteten Interventionen vor.

Unabhängig von der Angemessenheit und dem Wert des Placebo-Konzepts für Psychotherapiestudien sind zahlreiche Untersuchungen, in denen eine Vielzahl verschiedener psychologischer Placebo-Kontrollgruppen eingesetzt wurde, in der Forschungsliteratur zu finden. Während praktisch niemand die These infrage stellen würde, dass sich Psychotherapie im Vergleich zu verschiedenen unbehandelten Kontrollbedingungen als klinisch nützlich erwiesen hat, was die Möglichkeit einer Spontanremission bzw. Symptomverbesserung schlicht als Folge der Zeit ausschließt, widmete sich eine Welle der Psychotherapieforschung in den 1980erJahren dem relativen Nutzen von Psychotherapie im Vergleich zu einer Vielzahl unterschiedlicher Placebo-Kontrollen - trotz diverser Schwierigkeiten bei der Konzeption und praktischen Umsetzung der Studien sowie ethischer Bedenken. Der aktuelle Stand der Literatur zeigt Ergebnisse, die zu erwarten waren: Patienten in sogenannten Placebo-Kontrollgruppen weisen in der Regel eine stärkere Verbesserung auf als Patienten, die einer Wartelisten- oder unbehandelten Kontrollgruppe zugeordnet wurden [z.B. Barker et al., 1988; Bowers und Clum, 1988; Dush, 1986; Prioleau et al., 1983; Sheperd, 1984]. In einer groß angelegten Literaturstudie als Teil ihres umfangreichen Reviews von Meta-Analysen befassten sich Lipsey und Wilson [1993] mit dem Thema «Placebo». Die Befunde dieses Reviews legen nahe, dass theoriegeleitete Behandlungen sowohl einer unbehandelten Kontrollgruppe als auch einer Placebobehandlung deutlich überlegen sind. Sie schlussfolgern, dass «es sehr wahrscheinlich einige generalisierte Placeboeffekte gibt, die zu der Gesamtwirksamkeit einer psychologischen Behandlung beitragen, deren Stärke jedoch nicht ausreichend zu sein scheint, um in vollem Umfang diese Gesamteffekte zu erklären» (S. 1196-1197).

In einem anderen Review zum Placeboeffekt, das 46 meta-analytische Arbeiten einschloss, untersuchte Grissom [1996] Unterschiede zwischen Therapie- und Kontrollgruppe, Therapie und Placebo, Placebo und Kontrollgruppe sowie Therapie und einer anderen Therapie. Die Gesamtergebnisse «stimmen mit der Auffassung überein, dass sich in der Regel eine Rangfolge ergibt, nach der die Therapiebedingung am erfolgreichsten ist, gefolgt von Placebo und einer unbehandelten bzw. Wartelisten-Kontrollgruppe» (S. 979). Die Schätzung einer Placebo-Effektstärke (ES) von Lambert und Ogles [2004] ist nahezu identisch mit der von Lipsey und Wilson [1994] $(\mathrm{ES}=0,44)$ und der von Grissom [1996] $(\mathrm{ES}=0,48)$. Diese Daten legen nahe, dass der durchschnittliche placebobehandelte Patient im 60. Perzentil (anstatt im 50. Perzentil), und der durchschnittliche, mit Psychotherapie versorgte Patient im 80. Perzentil der Verteilung unbehandelter Kontrollprobanden liegt.

In der jüngeren Vergangenheit wurden zur Überwindung konzeptioneller und ethischer Schwierigkeiten Placebo-Kontrollgruppen weitgehend durch andere Kontrollbedingungen ersetzt. Neben solchen, die allgemeine Wirkfaktoren berücksichtigen, stehen alternativ Methoden wie z.B. vergleichende Behandlungsergebnisstudien zur Verfügung. Bei diesem Studiendesign werden zwei oder mehr Bona-fide-Behandlungen unter der Annahme gegenübergestellt, dass eine der Interventionen mehr wirksame, für den
Therapieerfolg notwendige Komponenten einschließt und daher $\mathrm{zu}$ einem positiven Ergebnis führt. Eine noch anspruchsvollere Forschungsstrategie umfasst Dismantling- und Add-on-Studiendesigns, bei denen Elemente effektiver Behandlungen isoliert werden und separat verabreicht werden, um ihren differenziellen Effekt sichtbar zu machen (d.h. welcher Aspekt der Behandlung kann ausgeklammert werden) [Borkovec, 1993].

Neben den bisher vorgestellten Forschungsansätzen gibt es einen weiteren, der versucht, spezifische Therapietechniken als Wirkfaktoren zu identifizieren, jedoch bisher ebenfalls eher enttäuschende Ergebnisse erbracht hat. Dieser Ansatz untersucht die Effektivität von mehr versus weniger geschulten Therapeuten. Es wäre anzunehmen, dass solche Vergleiche bessere Ergebnisse für höher qualifizierte Behandler zeigen und somit geschlussfolgert werden könnte, dass es von Bedeutung ist, Patienten mit theoriegeleiteten Therapietechniken zu behandeln, die im Mittelpunkt von Lehrveranstaltungen zu spezifischen Theorien und Verfahren der Psychotherapie und entsprechenden Praktikumserfahrungen stehen. Solche Studien erfassen indirekt den Effekt von - placeboartigen bzw. allgemeinen Wirkfaktoren gleichenden - Einflüssen der Vermittlung allgemeiner positiver interpersonaler Beziehungserfahrungen und einer authentischen Zuwendung und Unterstützung. Paraprofessionelle bzw. auszubildende Therapeuten, die bisher kaum in Kontakt mit spezifischen Therapietechniken standen, werden für ein solches Studienkonzept gezielt nach ihren interpersonellen Fähigkeiten ausgewählt [Stein und Lambert, 1995].

Studiendesigns mit Kontrollgruppen, die allgemeine Wirkfaktoren berücksichtigen, vergleichende Ergebnisstudien und Dismantling-Strategien zeigen in der Regel äquivalente Effekte für die Studienbedingungen und haben nur wenig dazu beigetragen, ursächliche Erklärungen für Therapieerfolge zu finden, mit Ausnahme der Schlussfolgerung, dass Therapieerfolg nicht ein einzigartiges Merkmal theoriegeleiteter Ansätze darstellt [Jacobson et al., 1996; Lambert, 2013; Wampold et al., 1997 (S. 205)]. Der Aufschluss aus diesen Studiendesigns über die ursächlichen Faktoren, nach denen so ernstlich über die letzten 50 Jahre experimenteller Ergebnisforschung gesucht wurde, ist somit nur gering. Außerhalb des Bereiches experimenteller Untersuchungen, in denen verschiedene Kontrollgruppen zum Einsatz kommen, könnten jedoch andere Befunde Licht ins Dunkel der Suche nach Kausalität bringen. Dazu zählen Erkenntnisse über die Terminierung des Zeitpunkts, zu dem Patienten auf eine Behandlung ansprechen - ein Thema von großer Bedeutung, wenn es um das Verständnis von Ursache-Wirkungs-Beziehungen in der Psychotherapie geht.

\section{Frühe Therapie-Response: Eine weitere Heraus- forderung für das Konzept theoriespezifischer Wirkkomponenten in der Psychotherapie}

In der pharmakologischen Forschung wird ein Medikament als wirksam angesehen, wenn sein Effekt sich von dem eines Placebos unterscheidet. In dieser Hinsicht ist häufig das Timing der Response ausschlaggebend für die Unterscheidung einer wirksamen phar- 
makologischen Substanz von einem Placebo. Von daher sind sowohl die langfristigen Effekte als auch der zeitliche Verlauf der Medikamentenwirkung wichtige Indikatoren für die Wirksamkeit. Ein frühes Ansprechen auf eine Behandlung kann eher eine vorzeitige Änderung in der Symptomatik bedingt durch den Akt der Einnahme eines vom Arzt verschriebenen Medikaments (z.B. die Erwartung des Patienten) bedeuten als die Wirkung des Präparats auf biologische Prozesse im Patienten. Diese «Placebo»-Reaktion wurde mit schlechteren Langzeitergebnissen - insbesondere mit Rückfällen während des Katamnesezeitraums - bei Patienten, die mit antidepressiven Medikamenten behandelt wurden, in Verbindung gebracht. Mehrere Studien haben dieses Phänomen untersucht und unterstützen das Argument, dass eine «vorzeitige» Response auf ein Medikament (eine Reaktion, die vor Verabreichung der therapeutischen Dosis erfolgt) schlechte therapeutische Langzeitergebnisse vorhersagt [Quitkin et al., 1984, 1987, 1996; Stewart, et al., 1998]. Abgesehen davon sieht die Medikamentenforschung jüngeren Datums vor, dass der Ausschluss von Patienten, die frühzeitig auf ein Placebo ansprechen, nicht erforderlich ist, da dadurch die Rückschlüsse auf die Wirksamkeit von Präparaten nicht verändert werden [Enck et al., 2013].

Es gibt nur wenige Studien, die das Timing der Response in einer Psychotherapie mit der Intention untersucht haben, die wirksamen Behandlungskomponenten und die Rolle allgemeiner sowie spezifischer therapeutischer Wirkfaktoren zu verstehen. In diesen Studien zeigt sich als konsistentes Ergebnis, dass ein frühes (im Gegensatz zu einem verzögerten) Ansprechen auf die Psychotherapie mit einem besseren mittel- und langfristigen Therapie-Outcome assoziiert ist - sprich, ein gegenteiliger Effekt zu dem, der in Arzneimittelstudien gefunden wurde. Als Beispiel untersuchten Fennel und Teasdale [1987] Patienten, die an einer Studie zur kognitiven Verhaltenstherapie (KVT) als Behandlung von Depressionen teilnahmen. Bei den Teilnehmern, die eine KVT erhielten, variierten die Behandlungsdosis, die anfängliche Therapie-Response der Patienten und das endgültige Behandlungsergebnis beträchtlich. Frühe Therapie-Responder schienen sich in einer anderen Art und Weise in die Therapie einzubringen als Patienten, die spät auf die Behandlung ansprachen, und hatten bessere Langzeitergebnisse nach Therapieabschluss. Beispielsweise schienen frühe TherapieResponder von einem Thema zum nächsten überzugehen (sequenzielles Verlaufsmuster), während verzögerte Responder das gleiche Therapiethema über die Therapiesitzungen hinweg immer wieder aufgriffen. Den Schlussfolgerungen der Autoren zufolge legt dies nahe, dass es im Zusammenhang mit einer frühen Therapie-Response anstelle eines vorübergehenden Placeboeffekts zu einer tiefgründigeren Auseinandersetzung und einem besseren Abschluss mit wichtigen therapeutischen Aufgaben kommt, was wiederum Voraussetzungen für therapieinduzierte Veränderungen sind (gegebenenfalls in Form einer Patientenvariable, nach der manche Patienten einfacher zu behandeln sind als andere, z.B. aufgrund einer höheren Therapiemotivation?).

In ähnlicher Weise untersuchten Renaud et al. [1988] ein schnelles Ansprechen auf die Therapie bei Jugendlichen, die wegen einer Depression behandelt wurden, und konnten zeigen, dass frühe Responder im Vergleich zu anfänglichen Non-Respondern bessere Ergebnisse zum Therapieende sowie zur 1- und 2-JahresKatamnese aufwiesen. Ein besonders wichtiges Ergebnis dieser Studie drehte sich um das eingesetzte Therapieverfahren. Es wurden drei Studienbedingungen miteinander verglichen: KVT, systemisch-behaviorale Familientherapie oder nichtdirektive supportive Therapie (NST). Die NST-Bedingung war dafür angedacht, «unspezifische Effekte» der Therapie bzw. die Präsenz therapeutenspezifischer sozialer Unterstützung zu kontrollieren. Aus diesem Grund beinhaltete die NST ein aktives Unterstützungsangebot, Affektklärung und aktives Zuhören. Im Gegensatz zu anfänglichen Non-Respondern wiesen frühe Responder unabhängig von der Behandlungsgruppe höhere Remissionsraten hinsichtlich ihrer klinischen Symptome auf; dieses Ergebnis war besonders drastisch in der NST-Bedingung (100\% vs. $21 \%$ Remissionsrate). Dieser Befund zeigte sich auch für die Einschätzung des Funktionsniveaus in der NST-Gruppe zur 2-Jahres Katamnese. Aus dieser sowie den im Folgenden diskutierten Studien geht hervor, dass zahlreiche Patienten bereits vor Behandlungsabschluss gut auf die Intervention ansprechen.

Illardi und Craighead [1994] führten ein Review zu Studien zur KVT bei Depressionen durch. Sie argumentierten, dass zeitliche Veränderungen im Rahmen der KVT (Response, die innerhalb der ersten 4 Therapiewochen beobachtet wurde) die Ansicht unterstützen, dass vorherrschend allgemeine Wirkfaktoren den Therapieerfolg in der KVT ursächlich erklären können. Sie wiesen darauf hin, dass die meisten Veränderungen in der KVT bereits auftraten, bevor spezifische KVT-Techniken - insbesondere die kognitive Umstrukturierungsstrategie - in einer therapeutischen Dosis zum Einsatz kamen. Des Weiteren argumentierten sie, dass - in Übereinstimmung mit Franks [1973] Konzept der Remoralisierung und Howards Phasenmodell der Psychotherapie [Kopta et al., 1994] dem Patienten Hoffnung vermittelt werden kann, indem ihm frühzeitig im Therapieverlauf das Therapierational erklärt wird und Hausaufgaben durchgeführt werden, was laut den Autoren allgemeine Wirkfaktoren darstellen. Eine frühe Therapie-Response kann schlichtweg ein Anzeichen für die Bereitschaft des Patienten sein, sich verändern zu wollen, im Zusammenspiel mit allgemeinen Wirkfaktoren anstatt spezifischen Interventionen (dies bedeutet, dass wesentliche Verbesserungen auftreten, bevor und nicht nachdem die meisten spezifischen therapeutischen Interventionen eingeleitet wurden).

Natürlich bieten diese Daten viel Interpretationsspielraum. Beispielsweise stimmen Tang und DeRubeis [1999] der Hypothese von Illardi und Craighead [1994] nicht zu und legen nahe, dass eine relativ hohe Dosis spezifischer Therapietechniken frühzeitig im Behandlungsverlauf zum Einsatz kam, da die KVT-Behandlungen in der Regel wöchentlich 2 Sitzungen innerhalb der ersten 4 Behandlungswochen umfassten. Wilson [1999], der mit Illardi und Craighead einer Meinung ist, wies darauf hin, dass sich in der Forschungsliteratur zur Behandlung von Bulimie und Alkoholmissbrauch ebenfalls Belege für die Annahme finden, dass frühzeitige (und relative große) Verbesserungen auftreten, bevor spezifische kognitive Techniken in der Therapie in therapeutischer Dosis um- 
gesetzt werden können. Solche Veränderungen in Patienten können nicht auf hypothetisch angenommene Wirkpfade kognitiver Interventionen zurückgeführt werden.

Eine solche Sichtweise wird durch Befunde zur BehandlungsResponse bei Zwangsstörungen untermauert. Heinzel und Schiepek [2014] untersuchten tägliche Messungen im therapeutischen Verlauf von 18 Zwangspatienten, die eine kognitiv-behaviorale Gruppenpsychotherapie einschließlich Exposition mit Reaktionsverhinderung erhielten. Sie zeigten, dass Symptomveränderungen bei vielen Patienten eher spontan auftraten und die Mehrheit dieser «Sudden Changes» (plötzlich auftretende, substanzielle Veränderungen im Therapieverlauf) direkt vor der Einleitung der Exposition mit Reaktionsverhinderung erfolgten. Darüber hinaus fanden sie, dass die «Sudden Gains» (plötzlich auftretende, substanzielle Verbesserungen im Therapieverlauf) möglicherweise in Verbindung mit Zuständen von Instabilität («dynamische Komplexität») stehen, die Verbesserungen vorausgehen. Heinzel und Schiepek [2014] spekulieren, dass therapeutische Aufgaben in Verbindung mit der Vorbereitung der Exposition mit Reaktionsverhinderung (wie z.B. das Unterschreiben eines Therapievertrags und dessen Präsentation in der Gruppe, das Planen und Durchführen kleinerer Konfrontationsübungen) Einfluss sowohl auf die Destabilisierung des Patienten wie auch auf die Symptomverbesserung haben. Sie sagen voraus, dass nicht erwartet werden kann, dass Veränderungen im Therapieprozess linear verlaufen, sondern vielmehr diskontinuierlich auftreten und mit Manifestationen kritischer Instabilitäten verbunden sind - einem Prozess der SelbstReorganisation und nicht der Exposition an sich.

Rief [2015] schlägt in einer persönlichen Mitteilung eine andere Erklärung vor: Die zeitliche Verknüpfung zwischen den Veränderungen und der Exposition «... weist darauf hin, dass die Sudden Changes nicht unabhängig von der Exposition erfolgen.» Entsprechend der Placebo-Perspektive von Rief lautet seine Erklärung folgendermaßen: «Den Patienten wird das Expositionsrational vermittelt; zunächst beängstigt sie dies möglicherweise, dann entwickeln sie aber die Hoffnung, auf dem richtigen Weg zu sein. Dies verstärkt positive Erwartungen hinsichtlich des Therapieergebnisses und führt zu einer Verbesserung, noch bevor die Exposition beginnt. Nach dieser Interpretation sind spezifische und allgemeine Wirkfaktoren nicht unabhängig voneinander, sondern interagieren miteinander (in beiden Richtungen).» Beide Interpretationsmöglichkeiten scheinen plausibel: Während Heinzel und Schiepek [2014] ihre Befunde auf der Grundlage ihrer Theorie der Selbst-Reorganisation interpretieren können, ist es ebenso nachvollziehbar, die Ergebnisse so zu erklären, dass die Bedeutung der spezifischen Technik der Exposition für den Therapieerfolg nicht infrage gestellt wird.

In einer anderen Studie untersuchten Aderka et al. [2011] Sudden Gains im Behandlungsverlauf und deren Beziehung zu kurzund langfristigen Therapie-Outcomes bei 91 Patienten mit Zwangsstörungen (Altersspanne: 19-64 Jahre), die entweder eine kognitive Therapie, eine Expositionsbehandlung oder eine Kombination aus einer der psychotherapeutischen Interventionen und Fluvoxamin durchliefen (insgesamt 4 Studienbedingungen). Die
Zwangssymptome der Teilnehmer wurden vor jeder wöchentlich stattfindenden Behandlungssitzung erhoben. Sudden Gains traten bei $34 \%$ der Teilnehmer auf und machten $66 \%$ der Gesamtreduktion der Zwangssymptome aus. Im Vergleich zu Patienten, die keine Sudden Gains erlebten, berichteten Teilnehmer, die plötzliche, substanzielle Verbesserungen im Therapieverlauf zeigten, geringer ausgeprägte Zwangssymptome zum Therapieabschluss und anhaltend bis zur Katamneseuntersuchung.

Angesichts der vorläufigen Befunde zur frühen Therapie-Response ist es wahrscheinlich verfrüht, die positiven Effekte nur einer der beiden Erklärungsmöglichkeiten - d.h. den spezifischen therapeutischen Interventionen oder den allgemeinen Wirkfaktoren zuzuschreiben. Es ist vernünftiger, daran zu erinnern, dass diese Erklärungsansätze nicht im Widerspruch zueinander stehen müssen, sondern vermutlich in komplexer Weise miteinander interagieren. Es besteht ein Bedarf an weiterer Forschung und verbesserter Methodik.

Derzeit mangelt es an Übereinstimmung in Bezug auf die Bestimmung und Messung von früher Behandlungs-Response. Man findet eine Vielzahl verschiedenartiger Definitionen von «early response»; u.a. verwendeten Fennel und Teasdale [1987] einen Mediansplit, um eine Responder-Rate zu bestimmen, während bei anderen Forscher ein einzelnes Item aus einem Fragebogen verwendet wurde. In einer Reihe von Studien wurde die Responder-Rate über das Ausmaß, in dem sich das Kliniker-Rating der Verbesserung/ Verschlechterung des Patienten von einer auf die nächste Woche veränderte, bestimmt [z.B. Quitkin et al., 1991]. Alternativ wurde das frühe Behandlungsansprechen darüber bestimmt, ob der Kliniker die Psychopathologie des Patienten nach der 1. oder 2. Behandlungswoche als gering ausgeprägt oder vollständig remittiert einstufte [Stewart et al., 1988]. Wieder andere Forscher kategorisierten jeden einzelnen Patienten im Hinblick auf seine Symptomreduktion und definierten dann die «early response» als eine Verbesserung von mehr als 50\% über eine vorgegebene Anzahl von Therapiesitzungen [Beckham, 1989; Renaud et al., 1998]. Nur wenige Studien haben die Response tatsächlich wöchentlich gemessen, weswegen es in den meisten Untersuchungen nicht gelungen ist, den Zeitpunkt (die Therapiesitzung) genau zu bestimmen, zu dem Patienten auf die Behandlung ansprechen. Tang und DeRubeis [1999] richteten ihr Interesse eher auf Sudden Gains, die über den gesamten Therapieverlauf und nicht nur frühzeitig auftreten, und haben für diese unter Anwendung des Beck-Depressions-Inventars spezifische Kriterien definiert.

Nachfolgende Studien, die weitgehend dieselben Kriterien wie Tang und DeRubeis [1999] verwendeten [Gaynor et al., 2003; Hardy et al., 2005; Hofmann et al., 2006; Stiles et al., 2003; Tang und DeRubeis, 1999b; Tang et al., 2005, 2002, 2007; Vittengl et al., 2005], zeigten ähnliche Ergebnisse. Plötzliche Veränderungen im Therapieverlauf wurden bei 17\% [Stiles et al., 2003] bis 50\% [Gaynor et al., 2003] der behandelten Patienten gefunden. In der Regel erklärten diese Sudden Gains mindestens 50\% aller Veränderungen über die gesamte Therapie; in allen bis auf eine Untersuchung [Stiles et al., 2003] schwankte die Varianzaufklärung innerhalb einer Spanne von 50-60\%. In einem Großteil der Studien zeigte 
sich eine signifikante Aufrechterhaltung des Therapieerfolgs über den Katamnesezeitraum. Zudem ergab sich über alle Untersuchungen hinweg die 5. Therapiesitzung als Median des Zeitpunkts, zu dem Sudden Gains auftraten. Studienübergreifend zeigte sich eine bemerkenswerte Konsistenz in den Befunden. Selbst in randomisiert kontrollierten Studien (RCTs) von 12-20 Sitzungen ergaben sich Sudden Gains häufig bereits sehr früh im Therapieverlauf, ironischerweise bevor der Großteil an therapeutischer Arbeit begann.

Eine alternative Definition von Sudden Gains wurde im Rahmen einer Studie von Haas et al. [2002] zur klinischen Routineversorgung basierend auf einer naturalistischen Datenbank (im Gegensatz zu einer RCT) operationalisiert und angewendet. Diese Forscher untersuchten das Therapie-Outcome von 147 Patienten mit vielfältigen affektiven, Angst- und Anpassungsstörungen, die mit unterschiedlichen monotherapeutischen und eklektischen Interventionen behandelt wurden. Die Patienten schätzten ihr psychisches Funktionsniveau wöchentlich ein. Weitere Erhebungen fanden im Zeitraum zwischen 6 Monaten bis 2 Jahren nach Therapieabschluss statt. Anstatt einen absoluten Wert zur Bestimmung von Sudden Gains festzulegen, entwickelten die Autoren eine relativ präzise, standardisierte Methode, um das Timing der Behandlungs-Response und ihrer Größe in Relation zu einer repräsentativen Response zu ermitteln. Daraus ergab sich für die Definition einer "early response» ein empirisch normativer statt eines stichprobenabhängigen Standards. Im Detail bedeutet dies, dass das Ausmaß bestimmt wurde, in dem sich jeder Patient zu einer bestimmten Therapiesitzung im Verhältnis zu einem Patienten mit demselben anfänglichen Wert veränderte. So wurde ein Sudden Gain basierend auf der Differenz zwischen einem durchschnittlichen Änderungswert bei Patienten (erwartete Veränderung) und der tatsächlichen Veränderung definiert, bereinigt um das Funktionsniveau zu Behandlungsbeginn und um die Anzahl der Therapiesitzungen. Im Vergleich zu Verfahren, die einen absoluten Standard festlegen, erlaubt dieses Vorgehen, die Regression zur Mitte zu berücksichtigen, indem davon ausgegangen wird, dass Patienten mit hohen Anfangswerten sich stärker verändern als Personen mit niedrigen Anfangswerten. Darüber hinaus werden Sudden Gains basierend auf einem Mittelwert über die ersten 3 Therapiesitzungen anstatt auf einem einzelnen Wert bestimmt. Damit stellt die Studie Behandlungsergebnisse von Patienten mit einer drastischen frühen (innerhalb der ersten 3 Sitzungen auftretenden) Behandlungs-Response dem Outcome von Patienten gegenüber, die kein Muster früher substanzieller Veränderungen im Therapieverlauf aufweisen.

Unter Verwendung dieses Verfahrens zeigen die Ergebnisse, dass 31\% (46/147) der Patienten die Kriterien einer «early dramatic response» erfüllen. Dieser Teil der Stichprobe, der substanzielle Verbesserungen frühzeitig in der Behandlung aufweist, macht $84 \%$ derjenigen Patienten aus, die sich entweder im Therapieverlauf verbessern oder bis zum Therapieende remittieren. Diejenigen hingegen, die langsamer und weniger drastisch auf die Therapie ansprechen, stellen die verbleibenden $16 \%$ der positiven Responder dar. Bei der Nachuntersuchung, durchschnittlich 1 Jahr nach Therapieabschluss, zeigte sich bei den frühen Respondern die Ten- denz, dass der Behandlungserfolg bestehen bleibt: 80\% der Patienten, die als verbessert oder remittiert zur Katamnese eingestuft wurden, wurden als «early dramatic responders» eingestuft.

Eine Replikation dieser Studien in einer ambulanten Ausbildungsklinik mit Patienten, die etwas schwerer gestört waren und die ebenfalls eine Vielzahl an Störungen aufwiesen, wurde von Johnson et al. [2001] durchgeführt. Ähnlich wie in der Studie von Haas et al. [2002] orientierten sich die Therapeuten an zahlreichen verschiedenen Therapieschulen und die Behandlung wurde außerhalb eines Forschungsprotokolls angeboten. Wie bei Haas et al. [2002] wurde die Behandlungs-Response mit dem Outcome Questionnaire (OQ)-45 im Verhältnis zu Abweichungen von normativen Response-Werten für verschiedene Stufen der Störungsausprägung zu Behandlungsbeginn gemessen. Darüber hinaus wurde eine «early response» als Reliable Change (Verbesserung um 14 oder mehr Punkte) zum Ende der 3. Behandlungssitzung definiert. Von den 75 Patienten wurden 19 (etwa 25\%) als frühe BehandlungsResponder klassifiziert.

Die Ergebnisse zeigten, dass - auch nach statistischer Kontrolle der anfänglichen Symptomausprägung - eine schnellere TherapieResponse zu einer stärkeren Verbesserung zum Therapieabschluss und zur Nachuntersuchung führte. Außerdem wurden frühe Therapie-Responder signifikant häufiger als «verbessert» zum Therapieende und zur 6-Monats-Katamnese eingestuft. Unter Verwendung der Kriterien für «early response» nach Haas et al. [2002] war das häufigste Ergebnis für «early responder» zum Therapieabschluss eine «klinisch signifikante Verbesserung» (in 14 von 19 Fällen, 74\%), während sich für die Non-Responder im frühen Therapieverlauf «keine Veränderung» als das häufigste Rating (in 41 von 56 Fällen, 73\%) ergab. Johnson et al. [2001] berichteten unter Verwendung der alternativen Definition von «early response» (Reliable Change nach Abschluss von 3 Behandlungssitzungen) über ähnliche Ergebnisse. Die Befunde der Katamnese zeigten die gleiche Tendenz, d.h. dass Patienten, die frühzeitig auf die Behandlung ansprechen, im Vergleich zu frühen Non-Respondern einen größeren Therapieerfolg haben und diesen aufrechterhalten können.

Die Befunde dieser beiden Studien [Haas et al., 2002; Johnson et al., 2001] stehen im Einklang mit Ergebnissen früherer Forschung zur psychologischen Behandlung von Depressionen [z.B. Fennel und Teasdale, 1987; Ranaud et al., 1998] und erweitern diese auf ambulant behandelte College-Studenten und Patienten in einer Krankenhausambulanz. Beide Stichproben schließen Patienten ein, die mit einer breiten Palette psychischer Probleme und Störungen und nicht allein einer Depression vorstellig wurden. Diese Ergebnisse stehen auch im Einklang mit der Behauptung von Illardi und Craidhead [1994], dass eine Veränderung in der Symptomatik weniger mit spezifischen und als wirksam vermuteten Therapiekomponenten, sondern eher mit unspezifischen und allgemeinen, therapieschulenübergreifenden Wirkfaktoren (z.B. Patient-Therapeut-Beziehung) zusammenhängt. Zugleich stehen die Ergebnisse von Haas et al. [2002] und Johnson et al. [2001] im Widerspruch zu denen von Tang und DeRubeis [1999], die besonders intensiv die Debatte über das Timing der Therapie-Response und dessen Auswirkungen auf allgemeine versus spezifische Wirkfaktoren 
führten. Dies kann möglicherweise darauf zurückgeführt werden, dass Tang und DeRubeis frühe Therapieabbrecher aus ihren Sudden Gains-Analysen ausschlossen und damit strenggenommen eine angemessene Untersuchung des Phänomens der frühen Behandlungs-Response in ihren Studien nicht möglich war.

Derzeit sind die Wirkmechanismen, die eine «early response» mit einem langfristigen Therapie-Outcome verknüpfen, unbekannt. Was auch immer die wirksamen Komponenten sind, sie scheinen in vielen Fällen schnell zu arbeiten. Der Zeitpunkt des Eintretens von Verbesserungen im Verlauf einer Psychotherapie hat theoretische Implikationen jenseits der placebobezogenen Erklärungen von Veränderungen. Wenn Patienten auf eine Therapie ansprechen, bevor die theoretisch bedeutsamen Techniken eingeleitet wurden, dann ist es schwierig, diesen allein eine zentrale Bedeutung im Heilungsprozess zuzuschreiben. Frühe Responder sind möglicherweise widerstandsfähiger, besser auf die Behandlung vorbereitet, motivierter und empfänglicher für therapeutische Einflüsse jeglicher Art. «Early response» kann auch ein besseres «Zusammenpassen» von Patient und Therapeut sowie positive Effekte der therapeutischen Arbeitsbeziehung widerspiegeln, die häufig zur 3. Behandlungssitzung nachgewiesen wurden.

\section{Schlussfolgerungen}

Der Einsatz von Placebo-Kontrollgruppen in Forschungsstudien hat gezeigt, dass viele sogenannte Placebos Einfluss auf das Wohlbefinden des Patienten haben, jedoch keinen zur Psychotherapie vergleichbaren Erfolg hervorrufen können. Gleichzeitig zeigen Forschungsbefunde, dass Verbesserungen in der Psychotherapie nicht in erster Linie auf der Wirkung spezifischer Techniken beruhen dies wurde anhand eines kurzen Reviews von Ergebnissen vergleichender Ergebnisstudien, Dismantling- und Add-on-Studien, Gegenüberstellungen von Therapieergebnissen geschulter versus ungeschulter Therapeuten und Studien zum Timing der BehandlungsResponse veranschaulicht. Angesichts des Anteils an Patienten, die eine «dramatic early treatment response» aufweisen, der Größe und langfristigen Stabilität ihres Effekts sowie ihres therapieschulenund störungsübergreifenden Auftretens sollten wir uns mehr mit ihrer Bedeutung beschäftigen. Evidenzbasierte Behandlungen und Behandlungsleitlinien gründen auf der Annahme, dass weitgehend spezifische therapeutische Techniken und Interventionen für die Verbesserung von Patienten verantwortlich sind, während sie Beweise dafür, dass andere Faktoren eine größere Rolle spielen, ignorieren. Zukünftige Forschung hinsichtlich des Timings der Behandlungs-Response und ihrer Ursachen wird als Weg zur weiteren Klärung von Wirkkomponenten der Psychotherapie empfohlen.

\section{Disclosure Statement}

Die Autoren erklären, dass kein Interessenkonflikt besteht, der die hier dargestellten Inhalte beeinflusst hat.

Übersetzt von Dr. Maria Kleinstäuber

\section{Literatur}

Aderka IM, Anholt GE, van Balkom AJ, Smit JH, Hermesh $\mathrm{H}$, van Oppen P: Sudden gains in the treatment of obsessive-compulsive disorder. Psychother Psychosom 2012;81:44-51.

Barker SL, Funk SC, Houston BK: Psychological treatment versus nonspecific factors: a meta-analysis of conditions that engender comparable expectations for improvement. Clin Psychol Rev 1988;8:579-594.

Beckham EE: Improvement after evaluation in psychotherapy of depression: evidence of a placebo effect? J Clin Psychol 1989;45:945-950.

Borkovec TD: Between-group therapy outcome research: design and methodology; in Onken LS, Blaine JD, Boren JJ (eds): Behavioral Treatments for Drug Abuse and Dependence. Rockville, National Institute on Drug Abuse, 1993, pp. 249-290.

Bowers T, Clum G: Relative contribution of specific and nonspecific treatment effects: meta-analysis of placebo-controlled behavior therapy research. Psychol Bull 1988;103:315-323.

Clerkin EM, Teachman BA, Smith-Janik SB: Sudden gains in group cognitive-behavioral therapy for panic disorder. Behav Res Ther 2008;46:1244-1250.

Critelli JW, Neumann KF: The placebo: conceptual analysis of a construct in transition. Am Psychol 1984;39: 32-39.

DeMarco CW: On the impossibility of placebo effects in psychotherapy. Philos Psychol 1998;11:207-227.
Dour HJ, Chorpita BF, Lee S, Weisz JR: Sudden gains as a long-term predictor of treatment improvement among children in community mental health organizations. Behav Res Ther 2013;51:564-572.

Dush DM: The placebo in psychosocial outcome evaluations. Eval Health Prof 1986;9:421-438.

Enck P, Bingel U, Schedlowski M, Rief W: The placebo re sponse in medicine: minimize, maximize or personalize? 2013;12:191-204.

Fennel MJV, Teasdale JD: Cognitive therapy for depression: individual differences and the process of change. Cognitive Ther Res 1987;11:253-271.

Frank JD: Persuasion and Healing. Baltimore, Johns Hop kins University Press, 1973

Gaynor ST, Weersing VR, Kolko DJ, Birmaher B, Heo J, Brent DA: The prevalence and impact of large sudden improvements during adolescent therapy for depression: a comparison across cognitive-behavioral, family, and supportive therapy. J Consult Clin Psychol 2003; 71:386-393.

Grissom RJ: The magical number.7 +/- .2: meta-metaanalysis of the probability of superior outcome in comparisons involving therapy, placebo, and control. J Consult Clin Psychol 1996;64:973-982.

Haas E, Hill R, Lambert MJ, Morrell B: Do early responders to psychotherapy maintain treatment gains? J Clin Psychol 2002;58:1157-1172.
Heinzel S, Schiepek G: Dynamic patterns in psychotherapy - discontinous changes and critical instabilities during the treatment of obsessive compulsive disorder. Nonlinear Dynamics Psychol Life Sci 2014;18:155-176.

- Hofmann SG, Schulz SM, Meuret AE, Moscovitch DA, Suvak M: Sudden gains during therapy of social phobia. J Consult Clin Psychol 2006;74:687-697.

Horvath P: Placebos and common factors in two decades of psychotherapy research. Psychol Bull 1988;104:214-225.

Ilardi SS, Craighead WE: The role of non-specific factors in cognitive-behavior therapy for depression. Clin Psychol Sci Prac 1994;1:138-156.

- Ilardi SS, Craighead WE: Commentary. Rapid early response, cognitive modification, and nonspecific factors in cognitive behavior therapy for depression: a reply to Tang and DeRubeis. Clin Psychol Sci Prac 1999;6: 295-299.

Ilardi SS, Craighead WE, Evans DD: Modeling relapse in unipolar depression: effects of dysfunctional cognitions and personality disorders. J Consult Clin Psychol 1997;65:381-391.

Jacobson NS, Dobson KS, Truax PA, Addis ME, Koerner K, Gollan JK, Gortner E, Prince SE: A component analysis of cognitive-behavioral treatment for depression. J Consult Clin Psychol 1996;64:295-304.

Johnson J, Lambert MJ, Sullivan E: Early Response and Psychotherapy Outcomes. Paper presented at the annual meetings of the American Psychological Association, San Francisco, 2001. 
Kopta SM, Howard KI, Lowry JL, Beutler LE: Patterns of symptomatic recovery in psychotherapy. J Consult Clin Psychol 1994;62:1009-1016.

Lambert MJ: The efficacy and effectiveness of psychotherapy; in Lambert MJ (ed): Bergin and Garfield's Handbook of Psychotherapy and Behavior Change, ed 6 . New York, Wiley and Sons, 2013.

Lambert MJ, Hansen NB, Umphress V, Lunnen K, Okiishi J, Burlingame GM, Reisinger CW: Administration and Scoring Manual for the OQ45.2. Stevenson, American Professional Credentialing Services, 1996.

Lambert MJ, Ogles BM: The efficacy and effectiveness of psychotherapy; in Lambert MJ (ed): Bergin and Garfield's Handbook of Psychotherapy and Behavior Change, ed 5. New York, Wiley and Sons, 2004, pp. 139-193.

Lipsey MW, Wilson DB: The efficacy of psychological, educational, and behavioral treatment: confirmation from meta-analysis. Am Psychol 1993;48:1181-1209.

Norton PJ, Klenck SC, Barrera TL: Sudden gains during cognitive-behavioral group therapy for anxiety disorders. J Anxiety Disord 2010;24:887-892.

Oei TPS, Shuttlewood GJ: Specific and nonspecific factors in psychotherapy: a case of cognitive therapy for depression. Clin Psychol Rev 1996;16:83-103.

Platt JR: Strong inference. Science 1964;147:347-353.

Prioleau L, Murdock M, Brody N: An analysis of psychotherapy versus placebo studies. Behav Brain Sci 1983;6: 275-310.
Quitkin FM, McGrath PJ, Stewart JW, Taylor BP, Klein DF: Can the effects of antidepressants be observed in the first two weeks of treatment? Neuropsychopharmacology 1996;15:390-394.

Quitkin FM, Rabkin JG, Markowitz J, Stewart JW, McGrath PJ, Harrison W: Use of pattern analysis to identify true drug response: a replication. Arch Gen Psychiatry 1987;44:259-264.

Quitkin FM, Rabkin JG, Ross D, McGrath PJ: Duration of antidepressant drug treatment: what is an adequate trial? Arch Gen Psychiatry 1984;41:238-245.

Quitkin FM, Rabkin JG, Stewart JW, McGrath PJ, Harrison W, Ross DC, Tricamo E, Fleiss J, Markowitz J, Klein DF: Heterogeneity of clinical response during placebo treatment. Am J Psychiatry 1991;148:193-196.

Renaud J, Brent DA, Baugher M, Birmaher B, Kolko DJ, Bridge J: Rapid response to psychosocial treatment for adolescent depression: a two-year follow-up. J Am Acad Child Adolesc Psychiatry 1998;37:1184-1191.

Rosenthal D, Frank JD: Psychotherapy and the placebo effect. Psychol Bull 1956;53:294-302.

Sheperd M: What price psychotherapy? BMJ 1984;288: 809-810.

Stein DM, Lambert MJ: Graduate training in psychotherapy: are therapy outcomes enhanced? J Consult Clin Psychol 1995;63:182-196.
Stewart JW, Quitkin FM, McGrath PJ, Amsterdam J, Fava M, Fawcett J, Reimherr F, Rosenbaum J, Beasley C Roback P: Use of pattern analysis to predict differential relapse of remitted patients with major depression during 1 year of treatment with fluoxetine or placebo. Arch Gen Psychiatry 1998;55:334-343.

Stiles WB, Leach C, Barkham M, Lucock M, Iveson S, Shapiro DA, Hardy GE: Early sudden gains in psychotherapy under routine clinic conditions: practice-based evidence. J Consult Clin Psychol 2003;71:14-21.

Tang TZ, DeRubeis RJ: Reconsidering rapid early response in cognitive behavioral therapy for depression. Clin Psychol Sci Prac 1999;6:283-288.

Tang TZ, Luborsky L, Andrusyna T: Sudden gains in recovering from depression: are they also found in psychotherapies other than cognitive-behavioral therapy? J Consult Clin Psychol 2002;70:444-447.

Vittengl JR, Clark LA, Jarrett RB: Validity of sudden gains in acute phase treatment of depression. J Consult Clin Psychol 2005;73:173-182.

Wampold BE, Mondin GW, Moody M, Stich F, Benson K, Ahn H: A meta-analysis of outcome studies comparing bona fide psychotherapies: empirically, 'all must have prizes'. Psychol Bull 1997;122:203-215.

Wilkins W: Psychotherapy: the powerful placebo. J Consult Clin Psychol 1984;52:570-573.

Wilson GT: Commentary. Rapid response to cognitive behavior therapy. Clin Psychol Sci Prac 1999;6:289-292. 